\title{
Uso de concentrados autólogos de plaquetas intraarticulares como coadyuvantes en el tratamiento quirúrgico de la rotura del ligamento cruzado anterior en una perra
}

\author{
Use of intra-articular autologous platelet concentrates as coadjutants in the surgical treatment \\ of a cranial cruciate ligament rupture in a bitch \\ RF Silva ${ }^{* a, b}$, CMF Rezende ${ }^{\mathrm{b}}$, JU Carmona ${ }^{\mathrm{a}}$ \\ ${ }^{a}$ Grupo de Investigación Terapia Regenerativa, Departamento de Salud Animal, Universidad de Caldas, Manizales, Colombia. \\ bEscola de Veterinária, Universidade Federal de Minas Gerais, Belo Horizonte, Minas Gerais, Brasil.
}

\begin{abstract}
SUMMARY
Cranial cruciate ligament rupture (CCLR) is one of the main orthopedic problems that could produce hind limb lameness in dogs. Even though this problem can be surgically treated the development and progression of osteoarthritis are typical features. A case of a dog that received postoperative intra-articular injections of autologous platelet concentrates (APCs) after the surgical reparation of a CCLR is described. The clinical, radiographic, and gait analysis results could potentially support the postoperative use of intra-articular injections of APCs as an adjunctive therapy in the CCLR surgical reparation.
\end{abstract}

Palabras clave: perro, rotura del ligamento cruzado anterior, plasma rico en plaquetas, terapia regenerativa.

Key words: dog, cranial cruciate ligament rupture, platelet rich plasma, regenerativ therapy.

\section{INTRODUCCIÓN}

La rotura del ligamento cruzado anterior (RLCA) es causa frecuente de cojera en perros (Johnson y col 1994, Vasseur 2003). Las técnicas quirúrgicas más utilizadas para tratar esta enfermedad son la estabilización extracapsular o intracapsular y técnicas modificadoras de la biomecánica de la articulación femoro-tibio-rotuliana (Kim y col 2008). Sin embargo, independientemente del tratamiento quirúrgico realizado, son frecuentes la impotencia funcional del miembro afectado y la aparición y progresión de osteoartritis (OA) de la articulación femoro-tibio-patelar (Elkins y col 1991, Hurley y col 2007).

Los concentrados autólogos de plaquetas (APCs) -también conocidos como plasma rico en plaquetas (PRP) - son una fuente autóloga de factores de crecimiento, principalmente factor de crecimiento transformante $\beta$ (TGF- $\beta$ ), factor de crecimiento derivado de las plaquetas (PDGF) y de otras proteínas que modulan la inflamación y la cicatrización (Carmona y col 2009). Los APCs han sido evaluados experimentalmente y han sido empleados clínicamente en diferentes campos de la medicina humana y veterinaria, con el fin de disminuir la inflamación y dolor posoperatorios, acelerar la cicatrización y producir la regeneración de los tejidos afectados (Carmona y Prades 2009).

Resultados recientes han indicado que los APCs pueden ser empleados de manera segura para el tratamiento de

Aceptado: 02.06.2011.

* Calle 65 Nº 26-10, Manizales, Colombia; raul.silva@ucaldas.edu.co artropatías en caballos (Carmona y col 2009) y seres humanos (Kon y col 2009, Filardo y col 2010). La inyección intraarticular de APCs reduce el dolor y mejora la función articular durante periodos de tiempo prolongados y con efecto superior al producido por terapia convencional (Carmona y col 2009). Solo se ha descrito el uso de APCs como coadyuvante en el manejo artroscópico de una lesión de osteocondritis disecante del hombro en un perro con excelentes resultados (Neumann y Viefhues 2010).

El objetivo de este artículo es describir el uso de APCs, obtenidos mediante el método del tubo (Silva y col 2011), como tratamiento coadyuvante en un caso de RLCA en un canino, que fue operado mediante técnica quirúrgica artroscópica para reemplazar el ligamento por injerto autólogo de fascia lata. Lo novedoso de este reporte estriba en los buenos resultados obtenidos en la deambulación en plataforma de fuerza y en el retraso en la progresión de cambios radiológicos degenerativos articulares en comparación con controles históricos.

\section{MATERIAL Y MÉTODOS}

\section{DESCRIPCIÓN DEL CASO CLÍNICO}

Una perra intacta SharPei de 19 meses de edad y con $19,5 \mathrm{~kg}$, fue evaluada por cojera unilateral del miembro posterior derecho con 7 días de duración, tras haber sufrido una caída desde una altura 1,5 m. En el examen clínico se detectaron positivas las pruebas del cajón anterior (con desplazamiento de $\sim 5 \mathrm{~mm}$ ) y de compresión tibial. Se realizó una artroscopia para confirmación diagnóstica 
de RLCA y tratamiento quirúrgico mediante reemplazo del ligamento cruzado anterior por injerto de fascia lata (Muzzi y col 2009). Después de la cirugía, la paciente recibió carprofeno $2,2 \mathrm{mg} / \mathrm{kg}$, cada 24 horas, y cefalexina $20 \mathrm{mg} / \mathrm{kg}$ cada 12 horas, ambos por vía oral, durante 7 días. Se realizó inyección intraarticular de APCs como tratamiento postoperatorio coadyuvante. La paciente fue evaluada en el tiempo para determinar la evolución de los cambios radiológicos asociados con la posible instauración y evolución de un cuadro de osteoartritis (OA) de rodilla y evaluación objetiva de marcha en plataforma de fuerza para determinar las fuerzas de apoyo verticales de cada miembro.

\section{OBTENCIÓN Y USO DE LOS CONCENTRADOS AUTÓLOGOS DE PLAQUETAS}

Los APCs fueron preparados de manera aséptica, según la técnica descrita por Silva y col (2011). Se obtuvo sangre de la paciente mediante venopunción safena con un catéter mariposa $21 \mathrm{G}$ (Shandong Weigao Group, China). La sangre fue depositada en 2 tubos de $8,5 \mathrm{~mL}$ con 1,5 mL de solución A de ACD (citrato de trisodio (22 g/L), ácido cítrico (8 g/L) y dextrosa $(24,5 \mathrm{~g} / \mathrm{L}))(\mathrm{BD}$, New Jersey, USA). La sangre fue centrifugada a $191 \mathrm{~g}$ durante 6 minutos. Con la ayuda de una aguja espinal $18 \mathrm{G}$ se recolectó $1 \mathrm{~mL}$ de plasma adyacente a la interfaz eritrocitos-plasma. Los APCs fueron analizados mediante hemograma automatizado (Abacus Junior Vet, Austria) y comparados contra los valores celulares de la sangre entera mediante una prueba $\mathrm{T}$ para muestras apareadas con un nivel de significancia del $95 \%$.

Tres dosis de $1,5 \mathrm{~mL}$ de APC fueron inyectadas vía intraarticular con un intervalo de dos semanas entre cada aplicación. La primera inyección fue efectuada al final del procedimiento quirúrgico. El volumen de APC aplicado fue igual a la cantidad de líquido sinovial retirado antes de la artroscopia. Los APCs fueron activados con gluconato de calcio $(-9,3 \mathrm{mg} / \mathrm{mL}-)$ (Ropsohn Therapeutics $\operatorname{Ltda}^{\circledR}$, Bogotá, Colombia) en una relación 10:1 antes de ser inyectados en la articulación.

\section{EVALUACIÓN RADIOGRÁFICA}

La evaluación radiográfica de la rodilla afectada se realizó antes de la cirugía y a los quince, treinta, sesenta, noventa y 150 días posoperatorios. La articulación femorotibio-rotuliana fue evaluada en las posiciones medio-lateral y craneocaudal. Los signos radiográficos de OA fueron analizados mediante la escala de Innes y col (2004), en la que se evalúan 4 parámetros: efusión articular (0-2), osteofitosis (0-3), mineralización intraarticular (0-2) y esclerosis subcondral tibial (0-1). La puntuación global de severidad de la enfermedad es obtenida mediante la suma y promedio de los valores de cada parámetro. En este sentido, 0 es normal y 3 es osteoartritis grave.

\section{EVALUACIÓN DE APOYO EN PLATAFORMA DE FUERZA}

La evaluación de la marcha (para determinar el grado objetivo de cojera -dolor-) fue evaluada con una plataforma de fuerza ubicada en una pista de marcha de 3 metros de longitud, $50 \mathrm{~cm}$ de ancho y $10 \mathrm{~cm}$ de altura. Las fuerzas verticales de reacción del suelo que actúan entre el miembro y la plataforma de fuerza fueron evaluadas en la fase de apoyo del ciclo de marcha. La paciente caminó a su propio ritmo (velocidad media 1,73 $\pm 0,44 \mathrm{~m} / \mathrm{s}$ ). La velocidad de marcha fue calculada con base en el tiempo de ciclo de la extremidad posterior izquierda, mediante la fórmula: velocidad = frecuencia (ciclos/s) x amplitud (m) (Bertram y Ruina 2001), para lo cual se utilizó una videocámara a $60 \mathrm{~Hz}$ (Sony DCR-SX63, China). Se evaluaron tres ciclos válidos por cada extremidad. Se consideró como ciclo válido el apoyo ipsilateral de los miembros. Los datos fueron adquiridos a una velocidad de $300 \mathrm{~Hz}$ y procesados con el programa DasyLab 10 (Measurement Computing, USA).

Los valores evaluados fueron el pico de fuerza vertical $\left(\mathrm{Pf}_{\mathrm{z}}\right)$ y el impulso vertical $\left(\mathrm{If}_{\mathrm{z}}\right)$. Estos fueron normalizados a la masa corporal (N/kg y N/kg/s, respectivamente). Cada variable se expresó como porcentaje del total de la fuerzaimpulso ejercido por todos los miembros mediante el uso de la fórmula: $\mathrm{X} \%_{(\text {miembro } A)}=\mathrm{F}_{(\text {miembro A })} /\left(\mathrm{F}_{\text {(miembro } A)}+\mathrm{F}_{\text {(miembro }}\right.$ B) $\left.+\mathrm{F}_{(\text {miembro C) }}+\mathrm{F}_{(\text {miembro } D)}\right)$ (Katic y col 2009), donde $\mathrm{X} \%$ representa el porcentaje de masa corporal distribuido a través del miembro; miembros A, B, C y D representan los cuatro miembros del perro y $\mathrm{F}$ representa el valor de fuerza-impulso evaluado del miembro correspondiente. Se calculó la media del valor de los tres ciclos de marcha por miembro para la normalización de las variables y se utilizó este valor para realizar los análisis. El análisis cinético fue realizado durante los mismos tiempos que fueron empleados para la evaluación radiográfica.

\section{RESULTADOS Y DISCUSIÓN}

La paciente no mostró ninguna complicación sistémica o local asociada con los sitios de incisión o con la inyección intraarticular de los APCs. Los APCs presentaron un conteo plaquetario estadísticamente $(\mathrm{P}=0,001)$ superior $(707,633 \pm 22,354$ plaquetas $/ \mu \mathrm{L})$, respecto a los conteos de plaquetas en sangre entera $(389,333 \pm 13,456$ plaquetas $/ \mu \mathrm{L})$. El conteo de leucocitos fue estadísticamente $(\mathrm{P}=0,001)$ inferior en los APCs $(9016 \pm 367$ células $/ \mu \mathrm{L})$ respecto al número de leucocitos presentes en sangre entera $(11,146 \pm 234$ células/ $\mu \mathrm{L})$. Estos valores concuerdan con los resultados para concentrar plaquetas caninas con el método del tubo (Silva y col 2011).

Los registros de la evaluación radiográfica mediante la escala de Innes y col (2004) fueron semejantes antes de la cirugía y a los 15, 30, 60 y 150 días posoperatorios (figura 1). En todos los momentos la puntuación de los parámetros radiográficos evaluados fue cero. Esto indica 


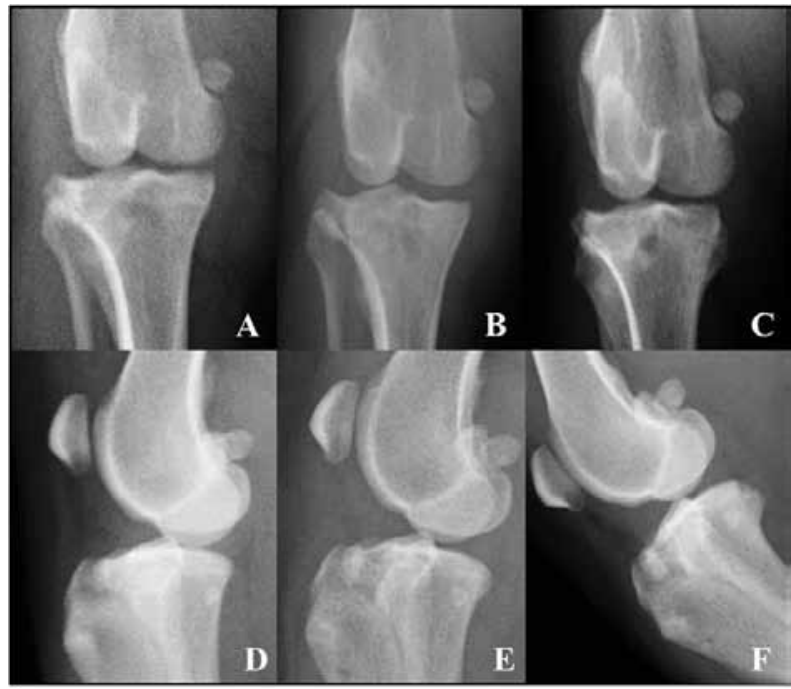

Figura 1. Radiografías craneocaudales (A, B y C) y medio laterales (D, E y F) de la paciente, antes (A y D) y a los 60 (B y E) y $150(\mathrm{C}$ y F $)$ días posoperatorios.

Cranio-caudal (A, B, and C) and medial-lateral (D, E, and $\mathrm{F})$ radiographs of the patient, before $(\mathrm{A}$ and $\mathrm{D})$ and 60 (B and $\mathrm{E})$ and $150(\mathrm{C}$ and $\mathrm{F})$ postoperative days.

que no se presentaron signos radiológicos de OA hasta el momento de haber tomado la última proyección (150 días posoperatorios).

La evaluación de la locomoción en la plataforma de fuerza indicó que los valores de $\mathrm{Pf}_{\mathrm{z}}$ e If $_{\mathrm{z}}$ comenzaron a homogeneizarse desde el $15^{\circ}$ día posoperatorio, hasta alcanzar finalmente valores muy iguales entre miembros contralaterales a los 90 días posoperatorios. Antes de la cirugía, la distribución porcentual de la masa corporal respecto a los valores de $\mathrm{Pf}_{\mathrm{z}}$ presentaron una distribución del 68\% hacia los miembros anteriores y del $25 \%$ hacia el miembro contralateral. Estos porcentajes de apoyo comenzaron a presentar un comportamiento homogéneo desde los 15 días posoperatorios y fueron muy similares entre los miembros contralaterales a los 90 días posoperatorios (cuadro 1).

Los resultados clínicos, radiográficos y cinemáticos observados en la paciente de este estudio fueron excelentes y por tal motivo los autores decidieron reportar el uso de APCs como tratamiento coadyuvante de la reparación quirúrgica de la RLCA en este caso. Las razones que hacen de este reporte un hecho relevante en clínica ortopédica canina son las siguientes:

1) Es poco habitual encontrar una articulación de rodilla canina reparada de RLCA sin ninguna evidencia radiológica de OA a los 150 días del posoperatorio. Estudios realizados en perros con RLCA que fueron tratados mediante técnicas intracapsulares han reportado de manera homogénea evidencia radiológica de $\mathrm{OA}$ a partir de las 4 semanas posoperatorias (Heffron y Campbell 1979, Elkins y col 1991, Vasseur y Berry 1992). Hurley y col (2007) encontraron evidencia radiológica de OA de rodilla 8 semanas después de haber realizado TPLO para
Cuadro 1. Valor medio de pico de fuerza vertical e impulso vertical en las cuatro extremidades y porcentaje de cada fuerza ejercida por cada miembro, antes del tratamiento quirúrgico y hasta 150 días posoperatorios.

Mean peak vertical force and vertical impulse in all the limbs and percentage of each force exerted by each limb, before surgery and up to 150 postoperative days.

\begin{tabular}{|c|c|c|c|c|c|c|}
\hline Variable & & empo (días) & MAD & MAI & MPD* & MPI \\
\hline \multirow{12}{*}{$\begin{array}{l}\mathrm{Pf}_{\mathrm{Z}} \\
\mathrm{N} / \mathrm{kg}\end{array}$} & \multirow{2}{*}{0} & Valor medio & 6,80 & 6,53 & 1,40 & 4,93 \\
\hline & & DMC \% & 34,6 & 33,2 & 7,1 & 25,1 \\
\hline & \multirow{2}{*}{15} & Valor medio & 7,54 & 8,51 & 3,45 & 7,30 \\
\hline & & DMC \% & 28,1 & 31,8 & 12,9 & 27,2 \\
\hline & \multirow{2}{*}{30} & Valor medio & 7,91 & 8,47 & 3,73 & 6,05 \\
\hline & & DMC \% & 30,2 & 32,4 & 14,3 & 23,1 \\
\hline & \multirow{2}{*}{60} & Valor medio & 5,94 & 6,38 & 3,85 & 4,39 \\
\hline & & DMC \% & 28,9 & 31,1 & 18,6 & 21,4 \\
\hline & \multirow{2}{*}{90} & Valor medio & 6,74 & 6,40 & 4,19 & 4,66 \\
\hline & & DMC \% & 30,6 & 29,1 & 19,1 & 21,2 \\
\hline & \multirow{2}{*}{150} & Valor medio & 7,62 & 7,16 & 5,30 & 5,40 \\
\hline & & DMC \% & 29,9 & 28,1 & 20,8 & 21,2 \\
\hline \multirow{12}{*}{$\begin{array}{l}\text { If }_{\mathrm{z}} \\
\mathrm{N} / \mathrm{kg} / \mathrm{s}\end{array}$} & \multirow{2}{*}{0} & Valor medio & 1,80 & 2,34 & 0,37 & 1,44 \\
\hline & & DMC \% & 30,3 & 39,3 & 6,2 & 24,2 \\
\hline & \multirow{2}{*}{15} & Valor medio & 1,41 & 1,37 & 0,48 & 0,99 \\
\hline & & DMC \% & 33,1 & 32,3 & 11,4 & 23,2 \\
\hline & \multirow{2}{*}{30} & Valor medio & 1,22 & 1,53 & 0,49 & 0,83 \\
\hline & & DMC $\%$ & 30,0 & 37,5 & 12,0 & 20,5 \\
\hline & \multirow{2}{*}{60} & Valor medio & 1,74 & 1,85 & 0,91 & 1,03 \\
\hline & & DMC \% & 31,5 & 33,4 & 16,5 & 18,6 \\
\hline & \multirow{2}{*}{90} & Valor medio & 1,65 & 1,81 & 0,93 & 1,01 \\
\hline & & DMC \% & 30,6 & 33,5 & 17,2 & 18,7 \\
\hline & \multirow{2}{*}{150} & Valor medio & 1,26 & 1,63 & 0,80 & 0,94 \\
\hline & & DMC \% & 27,2 & 35,3 & 17,2 & 20,3 \\
\hline
\end{tabular}

MAD: miembro anterior derecho, MAI: miembro anterior izquierdo, MPD: miembro posterior derecho, MPI: miembro posterior derecho. DMC: Distribución de masa corporal. *Miembro tratado.

corregir la misma enfermedad. Cabe aclarar que en esa investigación sólo fueron tomadas dos radiografías, una antes del tratamiento quirúrgico y otra 8 semanas después, por lo que no se descarta que los signos radiológicos de OA pudieron haberse presentado antes de la octava semana (Hurley y col 2007). Según la experiencia clínica de los autores con la técnica de reparación artroscópica (Muzzi y col 2009), es normal encontrar cambios osteoartríticos en las rodillas operadas a las 2 semanas posoperatorias. Por tal motivo, los autores consideran que en la paciente de este reporte la inyección intraarticular de APCs evitó (o al menos retrasó) la aparición de cambios osteoartríticos, al menos hasta los 150 días del posoperatorio. Sin embargo, este solo es un caso clínico aislado, por lo que no se puede generalizar a partir de estos resultados particulares.

2) Tanto las evaluaciones clínica (subjetiva) como cinética (objetiva) del grado de cojera mostraron una mejoría 
gradual de la marcha (locomoción) de la paciente al cabo de los 15 días posoperatorios y una mejoría completa (ausencia de cojera) después del primer mes posoperatorio. Esta mejoría se mantuvo hasta la última evaluación cinética a los 90 días posoperatorios. Los resultados observados en esta perra son esperanzadores puesto que en animales con RLCA tratados con TPLO, independientemente de recibir o no fisioterapia posoperatoria, pueden presentar cojera hasta 6 semanas posoperatorias (Monk y col 2006).

Lee y col (1999) afirman que bajo condiciones normales los miembros delanteros soportan el $60 \%$ de la masa corporal. También se ha reportado que en perros con marcha normal el porcentaje de distribución para cada miembro del $\mathrm{Pf}_{\mathrm{z}}$ es en promedio 58,9\% para los miembros anteriores y $41,1 \%$ para los miembros posteriores (Budsberg y col 1987), condición que la paciente de este reporte alcanzó de manera acelerada a los 15 días posoperatorios (cuadro 1). Trumble y col (2005) reportaron disminución drástica del $\mathrm{Pf}_{\mathrm{z}}$ dos semanas después del tratamiento quirúrgico extracapsular para RLCA. Estos resultados son totalmente opuestos a los encontrados en este reporte. Otro indicio importante que justifica el fuerte papel analgésico que cumplieron los APCs empleados como tratamiento coadyuvante intraarticular en la paciente de este reporte radica en el hecho de que pacientes operados con la misma técnica (con o sin fisioterapia posoperatoria) no lograron mejorar sus parámetros cinemáticos al cabo de 4 meses posoperatorios (Muzzi y col 2009).

3) Los resultados obtenidos en esta paciente indican el posible uso potencial de los APCs como un biofármaco con acción modificadora de la OA. Esta misma observación fue propuesta para caballos con AO que recibieron tratamiento intraarticular con APCs (Carmona y col 2009). Sin embargo, para confirmar o disuadir el uso de APCs como coayuvantes en la reparación de la RLCA en perros es necesario realizar estudios doble ciego aleatorizados y controlados para demostrar el potencial terapéutico de esta sustancia como coadyuvante en esta enfermedad.

\section{RESUMEN}

La ruptura del ligamento cruzado anterior (RLCA) es uno de los principales problemas ortopédicos que producen cojera de los miembros posteriores en perros. A pesar de que este problema sea tratado quirúrgicamente el desarrollo y progresión de la osteoartritis son típicamente característicos. Se describe un caso de un perro que recibió inyecciones intraarticulares de concentrados autólogos de plaquetas (APCs) durante el posoperatorio después de la reparación quirúrgica de una RLCA. Los resultados clínicos y radiográficos y el análisis de la marcha mediante plataforma de fuerza podrían potencialmente soportar la utilización posoperatoria de inyecciones intraarticulares de APCs como terapia coadyuvante en el tratamiento quirúrgico de la RLCA en perros.

\section{REFERENCIAS}

Bertram JEA, A Ruina. 2001. Multiple walking speed-frequency relations are predicted by constrained optimization. J Theor Biol 209, 445-453.
Budsberg SC, MC Verstraete, RW Soutas-Little. 1987. Force plate analysis of the walking gait in healthy dogs. Am J Vet Res 48, 915-918.

Carmona JU, C López, M Prades. 2009. Uso de concentrados autólogos de plaquetas obtenidos mediante el método del tubo como tratamiento de artropatías en caballos. Arch Med Vet 41, 175-179.

Carmona JU, M Prades. 2009. Platelet concentrates to treat musculoskeletal disease in horses. VDM Verlag, Saarbrücken, Germany.

Elkins AD, R Pechman, MT Kearney, M Herron. 1991. A retrospective study evaluating the degree of degenerative joint disease in the stifle joint of dogs following surgical repair of anterior cruciate ligament rupture. J Am Anim Hosp Assoc 27, 533-540.

Filardo G, E Kon, R Buda, A Timoncini, A Di Martino, A Cenacchi, PM Fornasari, S Giannini, M Marcacci. 2010. Platelet-rich plasma intra-articular knee injections for the treatment of degenerative cartilage lesions and osteoarthritis. Knee Surg Sports Traumatol Arthrosc DOI: 10.1007/s00167-010-1238-6.

Heffron LE, JR Campbell. 1979. Osteophyte formation in the canine stifle joint following treatment for rupture of the cranial cruciate ligament. J Small Anim Pract 20, 603-611

Hurley CR, DL Hammer, S Shott. 2007. Progression of radiographic evidence of osteoarthritis following tibial plateau leveling osteotomy in dogs with cranial cruciate ligament rupture: 295 cases (2001-2005). J Am Vet Med Assoc 230,1674-1679

Innes JF, M Costello, FJ Barr, H Rudorf, ARS Barr. 2004. Radiographic progression of osteoarthritis of the canine stifle joint: a prospective study. Vet Radiol Ultrasound 45, 143-148.

Johnson JA, C Austin, GJ Breur. 1994. Incidence of canine appendicular musculoskeletal disorders in 16 veterinary teaching hospitals from 1980 to 1989. Vet Comp Orthop Traumatol 7, 56-69.

Katic N, BA Bockstahler, M Mueller, C Peham. 2009. Fourier analysis of vertical ground reaction forces in dogs with unilateral hind limb lameness caused by degenerative disease of the hip jointand in dogs without lameness. Am J Vet Res 70, 118-126.

Kon E, R Buda, G Filardo, A Di Martino, A Timoncini, A Cenacchi, PM Fornasari, S Giannini, M Marcacci. 2009. Platelet-rich plasma: intra-articular knee injections produced favorable results on degenerative cartilage lesions. Knee Surg Sports Traumatol Arthrosc18, 472-479.

Kim SE, A Pozzi, MP Kowaleski, DD Levis. 2008. Tibial osteotomies for cranial cruciate ligament insufficiency in dogs. Vet Surg 37, 111-125.

Lee DV, JEA Bertram, RJ Todhunter. 1999. Acceleration and balance in trotting dogs. J Exp Biol 202, 3565-3573.

Monk ML, CA Preston, CM McGowan. 2006. Effects of early intensive posoperative physiotherapy on limb function after tibia plateau leveling osteotomy in dogs with deficiency of the cranial cruciate ligament. Am J Vet Res 67, 529-536.

Muzzi LAL, CMF Rezende, RAL Muzzi. 2009. Fisioterapia após substituição artroscópica do ligamento cruzado cranial em cães I-avaliação clínica, radiográfica e ultrassonográfica. Arq Bras Med Vet Zootec 61, 805-814

Neumann S, G Viefhues. 2010. Intraartikuläre Injektion von autologem Thrombozytenkonzentratbei der OCD. Veterinärspiegel 1, 22-26.

Silva RF, CMF Rezende, FO Paes-Leme, JU Carmona. 2011. Evaluación del método del tubo para concentrar plaquetas caninas: estudio celular. Arch Med Vet 43, 95-98.

Trumble TN, RC Billinghurst, AM Bendele, CW McIlwraith. 2005. Evaluation of changes in vertical ground reaction forces as indicators of meniscal damage after transection of the cranial cruciate ligament in dogs. Am J Vet Res 66, 156-163.

Vasseur PB. 2003. Stifle joint. In: Slatter D. Textbook of small animal surgery. $2^{\text {nd }}$ ed. Elsevier, Philadelphia, USA, Pp 2090-2133.

Vasseur PB, CR Berry. 1992. Progression of stifle osteoarthrosis following reconstruction of the cranial cruciate ligament in 21 dogs. J Am Anim Hosp Assoc 28,129-136. 